\title{
Elevated levels of PPAR-gamma in the cerebrospinal fluid of patients with multiple sclerosis
}

Levente Szalardy ${ }^{\mathrm{a}}$, Denes Zadori ${ }^{\mathrm{a}}$, Ervin Tanczos ${ }^{\mathrm{b}}$, Mihaela Simu ${ }^{\mathrm{c}}$, Krisztina Bencsik ${ }^{\mathrm{a}}$, Laszlo Vecsei ${ }^{\text {a,d }}$, Peter Klivenyi ${ }^{\text {a,* }}$

${ }^{\text {a }}$ Department of Neurology, University of Szeged, H-6725 Szeged, Semmelweis u. 6, Hungary

${ }^{b}$ Department of Mathematics and Computer Science, Eindhoven University of Technology, 5600 MB Eindhoven, P.O. Box 513, The Netherlands

${ }^{\mathrm{c}}$ Department of Neurology, University of Medicine and Pharmacy Victor Babes, 300736 Timisoara, Bd. Iosif Bulbuca nr. 10, Romania

${ }^{\mathrm{d}}$ Neuroscience Research Group of the Hungarian Academy of Sciences and the University of Szeged, H-6725 Szeged, Semmelweis u. 6, Hungary

Word count title: 13

Word count abstract: 218

Word count text: 1955

Word count legends: 105

Number of display items: 3 (1 table, 2 figures)

References: 38

Running header: Elevated PPAR-gamma levels in the cerebrospinal fluid of multiple sclerosis patients

* Corresponding author:

Peter Klivenyi, MD, PhD

Department of Neurology, University of Szeged, H-6725 Szeged, Semmelweis u. 6, Hungary

푱 Phone: +36-62-545-351; Fax: +36-62-545-497

E-mail: klivenyi.peter@med.u-szeged.hu 


\begin{abstract}
Peroxisome proliferator-activated receptor gamma (PPAR $\gamma)$, a ligand-activated transcriptional factor involved in the regulation of glucose and lipid metabolism, has gained interest as a potential therapeutic target in multiple sclerosis (MS) due to its potent immunoregulatory properties and the therapeutic efficacy of its ligands in experimental autoimmune encephalitis (EAE). Elevated expression of PPAR $\gamma$ has been observed in the spinal cord of EAE mice and in an in vitro model of antigen-induced demyelination; however, no reports have yet been available on the PPAR $\gamma$ status in the central nervous system of human individuals with MS. Aiming to identify a possible alteration, the present study assessed the levels of PPAR $\gamma$ protein in the cerebrospinal fluid (CSF) of MS patients via ELISA technique. We report a pronounced elevation in the CSF levels of PPAR $\gamma$ in MS patients $(n=35)$ compared to non-inflammatory controls $(n=22)$. This elevation was independent of blood-CSF barrier integrity, but correlated with CSF white blood cell count and IgG index, associating the observed elevation with neuroinflammation. Controlling for potential confounders, the CSF levels of PPAR $\gamma$ further displayed a moderate but significant association with clinical severity. Corroborating with prior experimental findings, these results may contribute to our understanding about the role of PPAR $\gamma$ in MS, and may implicate this protein as a potential CSF biomarker of the disease.
\end{abstract}

Key words: peroxisome proliferator-activated receptor gamma; multiple sclerosis; cerebrospinal fluid; ELISA; biomarker. 


\section{Introduction}

Peroxisome proliferator-activated receptors (PPARs) are members of a nuclear hormone receptor superfamily of ligand-activated transcriptional factors that also includes thyroid, steroid and retinoid receptors. Out of the three subtypes known (PPAR $\alpha, \operatorname{PPAR} \beta / \delta$ and PPAR $\gamma$ ), PPAR $\gamma$ has been the most extensively investigated. PPAR $\gamma$ is known to be critically involved in the regulation of glucose and lipid metabolism, but more recent results also revealed an important role of PPAR $\gamma$ in the regulation immune response [6], gathering an increasing research interest in the field of disorders involving autoimmune processes in the pathomechanism, such as multiple sclerosis (MS) [11, 27].

MS is a chronic debilitating inflammatory central nervous system (CNS) disorder, characterized by demyelination, axonal and neuronal degeneration. The disease is supposed to be of autoimmune origin, in which encephalitogenic T-helper 1 (Th1) and interleukin 17 (IL17)-producing Th17 lymphocytes are proposed to be the main mediators [1]. In the past years, natural and synthetic agonists of PPAR $\gamma$ were shown to effectively ameliorate the course of experimental autoimmune encephalitis (EAE), a widely used experimental model of MS, by inhibiting the expansion of encephalitogenic T cells [8, 9, 13, 21, 23, 29, 35]. More recently, the activation of PPAR $\gamma$ in both human and murine CD4+ $\mathrm{T}$ cells have been shown to potently inhibit the differentiation towards the Th17 lineage [16], and the eicozapentaenoic acid-induced amelioration of EAE has been reported to be accompanied by increased PPAR $\gamma$ in association with decreased IL-17 expression in CNS-infiltrating CD4+ T cells [35]. Conversely, pharmacological inhibition of $[28,29]$ or a heterozygous deficiency in PPAR $\gamma$ $[22,28,29]$ leads to an exacerbated course of EAE in mice in association with an augmented $\mathrm{T}$ cell response, suggesting altogether that the activation of the PPAR $\gamma$ pathway may be of 
therapeutic relevance in MS. Furthermore, elevated expression of PPAR $\gamma$ has been reported within the spinal cord of EAE mice [8,9] and in an in vitro model of antigen-induced demyelination [12], supporting the notion that PPAR $\gamma$ may have a physiological role in the regulation of CNS inflammation in rodents. However, no reports have yet been available on the PPAR $\gamma$ status in the CNS of human individuals with MS. This study aimed to identify a possible alteration of PPAR $\gamma$ expression in MS, by assessing the levels of PPAR $\gamma$ protein in the CSF of MS patients.

\section{Patients, materials and methods}

The study included CSF samples of 35 MS patients meeting the 2009 McDonald criteria for definite relapsing-remitting (RR) MS. Non-inflammatory control samples were collected from 22 age- and sex-matched patients whose differential diagnostic process necessitated a lumbar puncture (LP) but the results of which along with that of the imaging modalities revealed no abnormalities in the CNS. Written informed consent was obtained from all subjects and the study was approved by the local Ethical Committee at the University of Szeged. The demographic, clinical and laboratory parameters of the cohorts are summarized in Table 1. LPs were performed between 2010 and 2013. The patients were free of immunomodulatory therapy, and for patients experiencing a relapse $(n=27)$ LP was performed before the initiation of corticosteroid therapy.

Following the LP, CSF samples were centrifuged in $8.000 \mathrm{rpm}$ for 10 minutes. The supernatants were stored in sterile polypropylene tubes in $-80^{\circ} \mathrm{C}$ until use. Commercially available sandwich enzyme-linked immunosorbent assay (ELISA) kits were applied for the quantitative detection of PPAR $\gamma$, following the manufacturers' instruction (Uscn Life Science Inc., Wuhan, China). Samples and standards were run in duplicates. The optical density 
values were detected at 450/560 nm with a plate reader (Awareness Technology Inc, Palm City, FL, USA) and the respective concentrations were read from the standard curves fitted by Sigmaplot 10.0 (Systat Software Inc., Richmond, CA, USA). The intraassay and interassay coefficients of variation were $9 \%$ and $15 \%$, respectively. The analytical sensitivity of the assay is $51 \mathrm{pg} / \mathrm{ml}$; values below the detection limit (12/22 in the Control vs $4 / 35$ in the MS group) were interpolated from the standard curve to calculate median and provide measures for statistical computations. The CSF total protein concentration, the white blood cell count, the albumin quotient (CSF albumin / serum albumin) and the IgG index ((CSF IgG / serum $\mathrm{IgG})$ / albumin quotient) were determined in our diagnostic laboratory and were available in the clinical records. The Expanded Disability Status Scale (EDSS) score was evaluated for each patient. To provide standardised clinical measures, EDSS scores were comprehensively revised by a trained MS investigator (K.B.) who was blind to the experimental findings. The statistical analysis was performed by SPSS Statistics 17.0 (SPSS Inc., Chicago, IL, USA). The normality of data was assessed by Shapiro-Wilk test. Due to the non-Gaussian distribution of the obtained data in the MS cohort, non-parametric tests (Mann-Whitney U, Spearman's correlation) were used. Data in the text and in Table 1 are presented as median [interquartile range]. A $p<0.05$ value was regarded as significant; $p$ values of correlation analyses were corrected for multiple testing by the Bonferroni-Dunn method. Controlling for potential confounders, a binary logistic regression model and an ordinal regression model was established to assess the association of PPAR $\gamma$ with relapse and EDSS, respectively.

\section{Results}

In our study cohort, CSF levels of PPAR $\gamma$ were highly and significantly elevated in the CSF of patients with MS compared to Control (167.5 pg/ml [75.6-372.1 pg/ml] vs 40.4 pg/ml [16.5-73.1 pg/ml], $p<0.0005$, Fig. 1). The elevation of PPAR $\gamma$ appeared to be independent of 
blood-CSF barrier integrity as suggested by the lack of correlations with CSF total protein levels $($ Spearman's Rho $=0.110)$ and albumin quotient $($ Spearman's Rho $=-0.076)$ within the MS group. However, the CSF levels of PPAR $\gamma$ significantly and strongly correlated with the CSF cell count $(p<0.0005$, Spearman's Rho $=0.722$, Fig. 2$)$ and moderately correlated with the IgG index ( $p=0.007$, Spearman's Rho $=0.528$, Figure 2$)$. Though the highest levels of PPAR $\gamma$ were measured in MS patients experiencing a relapse at sampling and the median level of PPAR $\gamma$ was considerably higher among patients in relapse compared to those in remission (197.39 pg/ml [87.58-397.50 pg/ml] vs $112.98 \mathrm{pg} / \mathrm{ml}[67.76-245.07 \mathrm{pg} / \mathrm{ml}])$, CSF PPAR $\gamma$ level was not a significant predictor of relapse in a binary logistic regression model controlling for CSF cell count, total protein level, IgG index and albumin quotient as potential confounding covariates $(p=0.596, \operatorname{Exp}(\mathrm{B})=1.001,95 \%$ confidence interval $=0.997-1.005$, Wald $\mathrm{Chi}^{2}=0.281$, $\mathrm{df}=1$ ). Controlling for the same covariates in an ordinal regression model, CSF level of PPAR $\gamma$ displayed a moderate but significant $(p=0.011)$ positive association with EDSS with an estimate of $0.004(95 \%$ confidence interval $=0.001-0.007$; $\mathrm{df}$ $=1$ ), which calls for an odds ratio of 1.49 to have an increased EDSS by every $100 \mathrm{pg} / \mathrm{ml}$ elevation in the CSF concentration of PPAR $\gamma$.

\section{Discussion}

Our results demonstrate for the first time a pronounced elevation of PPAR $\gamma$ levels in the CSF of patients with MS, in association with increased CNS inflammatory parameters. These results are in line with the prior reports on elevated expression of PPAR $\gamma$ in the CNS of EAE mice $[8,9]$ and may reflect a possible physiological compensatory role of PPAR $\gamma$ in the regulation of CNS autoimmunity in humans. This experimentally demonstrated regulatory function is supposed to be primarily attributable to the attenuation of inflammatory cytokine expression within microglia/macrophages and astrocytes [30, 33, 37] and the (partly 
concomitant) suppression of the expansion of encephalitogenic Th1 [21], Th17 cells [16] and B lymphocytes [24], accompanied by a switch towards a Th2 response [10, 11]. In addition, activation of PPAR $\gamma$ expression in brain endothelial cells has been shown to be a negative regulator of lymphocytic transmigration [17]. Besides, PPAR $\gamma$ activation have also been demonstrated to have protective effects on neurons and oligodendrocytes $[2,10-12,20,26$, 34, 36]. Furthermore, the activation of PPAR $\gamma$ has been associated with the promotion of oligodendrocyte differentiation and myelin production $[3,7,15,25,31,34]$. These properties may all contribute to the positive effects of PPAR $\gamma$ activation in experimental CNS inflammation, and may be exploited in MS therapy. Based on the experimental findings, two small clinical studies have been performed with the synthetic agonist pioglitazone, the results of both supporting that the use of PPAR $\gamma$ agonists may be beneficial in MS [14, 32]. Moreover, in a population-based study, the Pro12Ala polymorphism in the PPARG gene strongly associated with a delayed clinical onset of MS, which draws a further link between MS and PPAR $\gamma$ function [19].

The source of the elevated levels of PPAR $\gamma$ observed in this study, however, is not entirely clear, and its determination will require further investigations. It is known that PPAR $\gamma$ is expressed in neurons, oligodendrocytes, astrocytes, microglia/macrophages [3], $\mathrm{T}$ and $\mathrm{B}$ lymphocytes, dendritic cells [38] and brain endothelial cells [17]. In a murine EAE model of MS an increased expression of PPAR $\gamma$ has been reported in spinal cord homogenates [9] and in microglia/macrophages and astrocytes within the spinal cord lesions [8]. Furthermore, a strong upregulation of PPAR $\gamma$ has been observed in three-dimensional rat brain cultures following demyelination induced by antibodies directed against myelin oligodendrocyte glycoprotein [12]. Considering the neuropathological alterations in MS, the above listed experimental findings and our observation that PPAR $\gamma$ levels correlated with CSF inflammatory parameters, it is possible that PPAR $\gamma$ protein leaking out from 
injured/degenerating oligodendrocytes, neurons and astrocytes contribute to the observed elevation of its levels in the CSF. Considering that PPAR $\gamma$ upregulation has been demonstrated in activated macrophages [30] and activated $\mathrm{T}$ lymphocytes $[4,5]$, and that PPAR $\gamma$ levels correlated with the CSF cell count in our study, the contribution of such cellular origin to the observed phenomenon may not be fully excluded either; however, the fact that our CSF samples were centrifuged almost immediately after LP suggest that the extent of a putative cell disintegration within the obtained sample should most probably be insufficient to cause such a significant alteration in the concentration of PPAR $\gamma$ in the supernatant. Though future investigations are necessary to conclude on the exact source of the observed elevation, the possible sources discussed - in light with the findings of prior experimental studies suggest that the level of PPAR $\gamma$ in the CSF may reflect the severity of CNS involvement, which could account for the observed positive association with EDSS in our study.

The literature regarding alterations in PPAR $\gamma$ status in MS is rather sparse. Somewhat contradicting to our findings, another small clinical study examining peripheral white blood cells of MS patients reported decreased expression of PPAR $\gamma$ compared to control [18]. The findings of the two studies, however, are hard to compare, given that the studies focus on different compartments; furthermore, the patients enrolled in that study were either under immunomodulatory therapy or already in a secondary progressive phase, most probably representing different conditions compared to our untreated RR patients.

\section{Conclusions}

CSF levels of PPAR $\gamma$ were pronouncedly elevated in MS patients. This elevation was independent of blood-CSF barrier integrity, strongly correlated with white blood cell count, moderately correlated with IgG index, and displayed a moderate association with clinical 
severity. These results may contribute to our understanding about the role of PPAR $\gamma$ in the pathogenesis MS, and implicate this protein as a potential CSF biomarker of the disease. Further research is necessary to determine the exact background of the observed alteration, and to assess the potential clinical relevance of this novel observation.

\section{Acknowledgements}

The study was supported by the European Regional Development Fund HURO/0901/021/2.2.3 by the frame-work of the Hungary-Romania Cross-border Cooperation Programme 2007-2013. The authors' research activities are supported by the

projects OTKA (K 75628), TÁMOP-4.2.2/B-10/1-2010-0012 and TÁMOP-4.2.2.A11/1/KONV-2012-0052. We are grateful for Agnes Koszo, Gabriella Keszegne Voros and Andrea Toth for the excellent technical assistance.

\section{Conflicts of interest}

The authors report no conflicts of interest. 


\section{References}

1 A. Amedei, D. Prisco, M.M. D'Elios, Multiple sclerosis: the role of cytokines in pathogenesis and in therapies, Int. J. Mol. Sci. 13 (2012) 13438-13460.

2 V. Benedusi, F. Martorana, L. Brambilla, A. Maggi, D. Rossi, The peroxisome proliferator-activated receptor gamma (PPARgamma) controls natural protective mechanisms against lipid peroxidation in amyotrophic lateral sclerosis, J. Biol. Chem. 287 (2012) 35899-35911.

3 A. Bernardo, L. Minghetti, Regulation of Glial Cell Functions by PPAR-gamma Natural and Synthetic Agonists, PPAR Res. 2008 (2008) 864140.

4 R.B. Clark, D. Bishop-Bailey, T. Estrada-Hernandez, T. Hla, L. Puddington, S.J. Padula, The nuclear receptor PPAR gamma and immunoregulation: PPAR gamma mediates inhibition of helper T cell responses, J. Immunol. 164 (2000) 1364-1371.

5 R. Cunard, M. Ricote, D. DiCampli, D.C. Archer, D.A. Kahn, C.K. Glass, C.J. Kelly. Regulation of cytokine expression by ligands of peroxisome proliferator activated receptors, J. Immunol. 168 (2002) 2795-2802.

6 R.A. Daynes, D.C. Jones, Emerging roles of PPARs in inflammation and immunity, Nat. Rev. Immunol. 2 (2002) 748-759.

7 C. De Nuccio, A. Bernardo, R. De Simone, E. Mancuso, V. Magnaghi, S. Visentin, L. Minghetti, Peroxisome proliferator-activated receptor gamma agonists accelerate oligodendrocyte maturation and influence mitochondrial functions and oscillatory $\mathrm{Ca}(2+)$ waves, J. Neuropathol. Exp. Neurol. 70 (2011) 900-912.

8 A. Diab, C. Deng, J.D. Smith, R.Z. Hussain, B. Phanavanh, A.E. Lovett-Racke, P.D. Drew, M.K. Racke, Peroxisome proliferator-activated receptor-gamma agonist 15deoxy-Delta(12,14)-prostaglandin $\mathrm{J}(2)$ ameliorates experimental autoimmune encephalomyelitis, J. Immunol. 168 (2002) 2508-2515.

9 A. Diab, R.Z. Hussain, A.E. Lovett-Racke, J.A. Chavis, P.D. Drew, M.K. Racke, Ligands for the peroxisome proliferator-activated receptor-gamma and the retinoid $\mathrm{X}$ receptor exert additive anti-inflammatory effects on experimental autoimmune encephalomyelitis, J. Neuroimmunol. 148 (2004) 116-126.

10 P.D. Drew, P.D. Storer, J. Xu, and J.A. Chavis, Hormone regulation of microglial cell activation: relevance to multiple sclerosis, Brain Res. Brain Res. Rev. 48 (2005) 322327.

11 P.D. Drew, J. Xu, M.K. Racke, PPAR-gamma: Therapeutic Potential for Multiple Sclerosis, PPAR Res. 2008 (2008) 627463.

12 C.B. Duvanel, P. Honegger, H. Pershadsingh, D. Feinstein, J.M. Matthieu, Inhibition of glial cell proinflammatory activities by peroxisome proliferator-activated receptor gamma agonist confers partial protection during antimyelin oligodendrocyte glycoprotein demyelination in vitro, J. Neurosci. Res. 71 (2003) 246-255.

13 D.L. Feinstein, E. Galea, V. Gavrilyuk, C.F. Brosnan, C.C. Whitacre, L. DumitrescuOzimek, G.E. Landreth, H.A. Pershadsingh, G. Weinberg, M.T. Heneka, Peroxisome proliferator-activated receptor-gamma agonists prevent experimental autoimmune encephalomyelitis, Ann. Neurol. 51 (2002) 694-702.

14 C.C. Kaiser, D.K. Shukla, G.T. Stebbins, D.D. Skias, D.R. Jeffery, D. Stefoski, G. Katsamakis, D.L. Feinstein, A pilot test of pioglitazone as an add-on in patients with relapsing remitting multiple sclerosis, J. Neuroimmunol. 211 (2009) 124-130.

15 S. Kanakasabai, E. Pestereva, W. Chearwae, S.K. Gupta, S. Ansari, J.J. Bright, PPARgamma agonists promote oligodendrocyte differentiation of neural stem cells by modulating stemness and differentiation genes, PLoS One, 7 (2012) e50500. 
16 L. Klotz, S. Burgdorf, I. Dani, K. Saijo, J. Flossdorf, S. Hucke, J. Alferink, N. Nowak, M. Beyer, G. Mayer, B. Langhans, T. Klockgether, A. Waisman, G. Eberl, J. Schultze, M. Famulok, W. Kolanus, C. Glass, C. Kurts, P.A. Knolle, The nuclear receptor PPAR gamma selectively inhibits Th17 differentiation in a $\mathrm{T}$ cell-intrinsic fashion and suppresses CNS autoimmunity, J. Exp. Med. 206 (2009) 2079-2089.

17 L. Klotz, L. Diehl, I. Dani, H. Neumann, N. von Oppen, A. Dolf, E. Endl, T. Klockgether, B. Engelhardt, B. Knolle, Brain endothelial PPARgamma controls inflammation-induced $\mathrm{CD} 4+\mathrm{T}$ cell adhesion and transmigration in vitro, $\mathrm{J}$. Neuroimmunol. 190 (2007) 34-43.

18 L. Klotz, M. Schmidt, T. Giese, M. Sastre, P. Knolle, T. Klockgether, M.T. Heneka, Proinflammatory stimulation and pioglitazone treatment regulate peroxisome proliferator-activated receptor gamma levels in peripheral blood mononuclear cells from healthy controls and multiple sclerosis patients, J. Immunol. 175 (2005) 49484955.

19 L. Klotz, S. Schmidt, R. Heun, T. Klockgether, H. Kolsch, Association of the PPARgamma gene polymorphism Pro12Ala with delayed onset of multiple sclerosis, Neurosci. Lett. 449 (2009) 81-83.

20 R. Luna-Medina, M. Cortes-Canteli, M. Alonso, A. Santos, A. Martinez, A. PerezCastillo, Regulation of inflammatory response in neural cells in vitro by thiadiazolidinones derivatives through peroxisome proliferator-activated receptor gamma activation, J. Biol. Chem. 280 (2005) 21453-21462.

21 C. Natarajan, J.J. Bright, Peroxisome proliferator-activated receptor-gamma agonists inhibit experimental allergic encephalomyelitis by blocking IL-12 production, IL-12 signaling and Th1 differentiation, Genes Immun. 3 (2002) 59-70.

22 C. Natarajan, G. Muthian, Y. Barak, R.M. Evans, J.J. Bright, Peroxisome proliferatoractivated receptor-gamma-deficient heterozygous mice develop an exacerbated neural antigen-induced Th1 response and experimental allergic encephalomyelitis, J. Immunol.171 (2003) 5743-5750.

23 M. Niino, K. Iwabuchi, S. Kikuchi, M. Ato, T. Morohashi, A. Ogata, K. Tashiro, K. Onoe, Amelioration of experimental autoimmune encephalomyelitis in C57BL/6 mice by an agonist of peroxisome proliferator-activated receptor-gamma, J. Neuroimmunol. 116 (2001) 40-48.

24 J. Padilla, E. Leung, R.P. Phipps, Human B lymphocytes and B lymphomas express PPAR-gamma and are killed by PPAR-gamma agonists, Clin. Immunol. 103 (2002) 22-33.

25 A.S. Paintlia, M.K. Paintlia, A.K. Singh, J.K. Orak, I. Singh, Activation of PPARgamma and PTEN cascade participates in lovastatin-mediated accelerated differentiation of oligodendrocyte progenitor cells, Glia 58 (2010) 1669-85.

26 A.S. Paintlia, M.K. Paintlia, I. Singh, A.K. Singh, IL-4-induced peroxisome proliferator-activated receptor gamma activation inhibits NF-kappaB trans activation in central nervous system (CNS) glial cells and protects oligodendrocyte progenitors under neuroinflammatory disease conditions: implication for CNS-demyelinating diseases, J. Immunol. 176 (2006) 4385-4398.

27 M.K. Racke, A.R. Gocke, M. Muir, A. Diab, P.D. Drew, A.E. Lovett-Racke, Nuclear receptors and autoimmune disease: the potential of PPAR agonists to treat multiple sclerosis, J. Nutr. 136 (2006) 700-703.

28 H.P. Raikwar, G. Muthian, J. Rajasingh, C. Johnson, J.J. Bright, PPARgamma antagonists exacerbate neural antigen-specific Th1 response and experimental allergic encephalomyelitis, J. Neuroimmunol. 167 (2005) 99-107. 
29 H.P. Raikwar, G. Muthian, J. Rajasingh, C.N. Johnson, J.J. Bright, PPARgamma antagonists reverse the inhibition of neural antigen-specific Th1 response and experimental allergic encephalomyelitis by Ciglitazone and 15-deoxy-Delta12,14prostaglandin J2, J. Neuroimmunol. 178 (2006) 76-86.

30 M. Ricote, A.C. Li, T.M. Willson, C.J. Kelly, C.K. Glass, The peroxisome proliferator-activated receptor-gamma is a negative regulator of macrophage activation, Nature 391 (1998) 79-82.

31 A.D. Roth, A.V. Leisewitz, J.E. Jung, P. Cassina, L. Barbeito, N.C. Inestrosa, M. Bronfman, PPAR gamma activators induce growth arrest and process extension in B12 oligodendrocyte-like cells and terminal differentiation of cultured oligodendrocytes, J. Neurosci. Res. 72 (2003) 425-435.

32 D.K. Shukla, C.C. Kaiser, G.T. Stebbins, D.L. Feinstein, Effects of pioglitazone on diffusion tensor imaging indices in multiple sclerosis patients, Neurosci. Lett. 472 (2010) 153-6.

33 P.D. Storer, J. Xu, J. Chavis, P.D. Drew, Peroxisome proliferator-activated receptorgamma agonists inhibit the activation of microglia and astrocytes: implications for multiple sclerosis, J. Neuroimmunol. 161 (2005) 113-122.

34 C.R. Swanson, V. Joers, V. Bondarenko, K. Brunner, H.A. Simmons, T.E. Ziegler, J.W. Kemnitz, J.A. Johnson, M.E. Emborg, The PPAR-gamma agonist pioglitazone modulates inflammation and induces neuroprotection in parkinsonian monkeys, J. Neuroinflammation 8 (2011) 91.

35 K. Unoda, Y. Doi, H. Nakajima, K. Yamane, T. Hosokawa, S. Ishida, F. Kimura, T. Hanafusa, Eicosapentaenoic acid (EPA) induces peroxisome proliferator-activated receptors and ameliorates experimental autoimmune encephalomyelitis, J. Neuroimmunol. 256 (2013) 7-12.

36 B. Xing, T. Xin, R.L. Hunter, G. Bing, Pioglitazone inhibition of lipopolysaccharideinduced nitric oxide synthase is associated with altered activity of p38 MAP kinase and PI3K/Akt, J. Neuroinflammation 5 (2008) 4.

37 J. Xu, P.D. Drew, Peroxisome proliferator-activated receptor-gamma agonists suppress the production of IL-12 family cytokines by activated glia, J. Immunol. 178 (2007) 1904-1913.

38 Y. Yang, A.E. Lovett-Racke, M.K. Racke, Regulation of Immune Responses and Autoimmune Encephalomyelitis by PPARs, PPAR Res. 2010 (2010) 104705. 


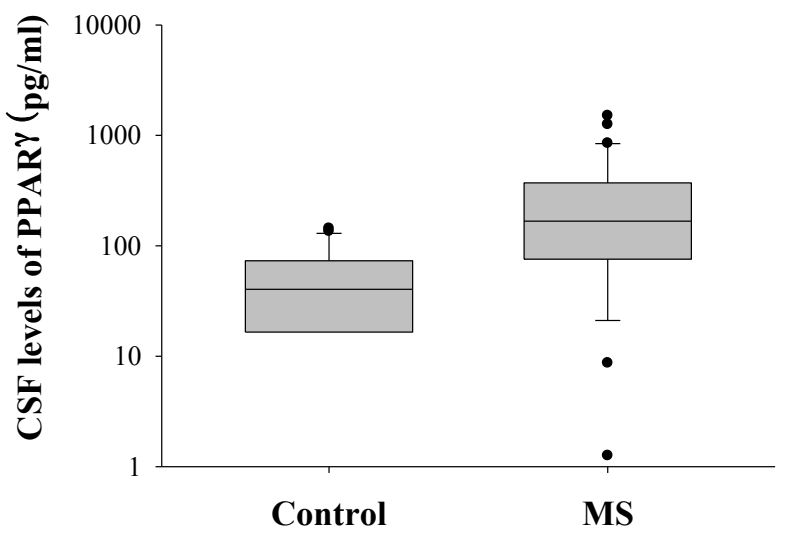

Fig. 1. PPAR $\gamma$ concentrations are elevated $(p<0.0005)$ in the CSF of patients with MS $(\mathrm{n}=35)$ compared to control $(\mathrm{n}=22)$. The box-plots present median and quartile values. Abbrevations: CSF, cerebrospinal fluid; MS, multiple sclerosis; PPAR $\gamma$, peroxisome proliferator-activated receptor gamma. 

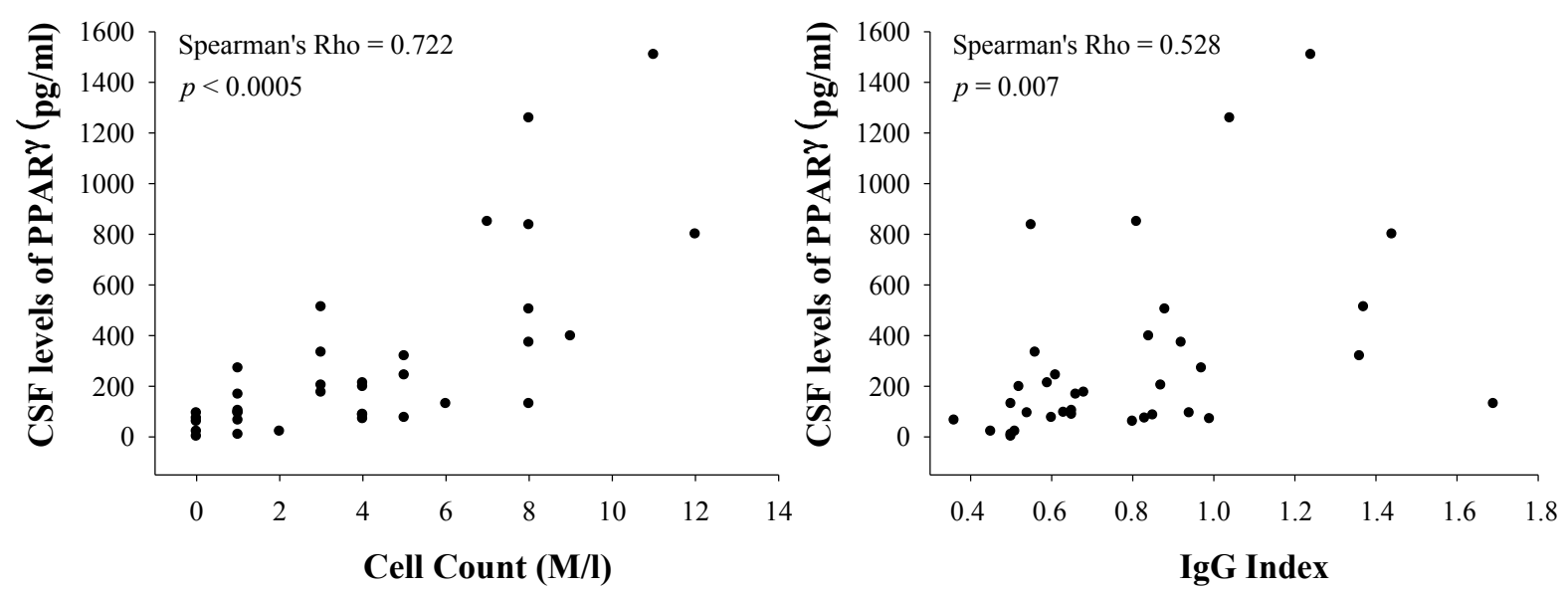

Fig. 2. PPAR $\gamma$ levels correlate with white blood cell count and IgG index in the CSF of patients with MS. Abbrevations: CSF, cerebrospinal fluid; MS, multiple sclerosis; PPAR $\gamma$, peroxisome proliferator-activated receptor gamma. 
Control

22

No. of subjects

Female/male

$\begin{array}{cc}\text { Age } & \text { year } \\ \text { Cell count } & \mathrm{M} / 1 \\ \text { Total protein } & \mathrm{mg} / 1 \\ \text { Albumin quotient } & \mathrm{x} \mathrm{10^{3 }} \\ \text { IgG index } & \end{array}$

Relapse/remission

EDSS

Disease duration

2

$13 / 9$

$10^{3}$

month
$34.0[26.2-47.3]$

34.3 [27.5-42.1]

0.660

$$
1.0[0.0-1.3]
$$

$4.0[1.0-7.0]$

0.001

325.0 [250.0-403.0]

400.0 [341.0-510.0]

0.007

$6.0[5.0-7.0]$

$0.7[0.6-0.9]$

$27 / 8$

$2.5[2.0-3.0]$

$18[6.0-36.0]$

Table 1. Demographic, laboratory and clinical parameters of the subject cohorts. The measured data are presented as median [interquartile range]. Abbrevations: EDSS, expanded disability status scale; MS, multiple sclerosis. 